\title{
Research on Structure Design of Dynamic Hydrocyclone on Board Ship
}

\author{
Cheng Xiang-xin*, Zhang Qiang, Wu Shuo, Li De-yu \\ Naval Architecture \& Marine Engineering College, Shandong Jiaotong University, Weihai, China
}

Email address:

chengxiangxin@sina.com (Cheng Xiang-xin)

${ }^{*}$ Corresponding author

To cite this article:

Cheng Xiang-xin, Zhang Qiang, Wu Shuo, Li De-yu. Research on Structure Design of Dynamic Hydrocyclone on Board Ship. American Journal of Mechanics and Applications. Vol. 4, No. 1, 2016, pp. 25-29. doi: 10.11648/j.ajma.20160401.15

Received: October 24, 2016; Accepted: November 15, 2016; Published: December 14, 2016

\begin{abstract}
Treatment about bilge water is always one key point of the ship pollution prevention, at present, separation accuracy of the separator on board can reach requirements of specification for sea-going ships pollution prevention, but the separation of quantity and convenience of operation aspects are still to be improved. The ship oily water dynamic hydrocyclone separator can both meet to the above two aspects. The thinking of structure design and the manufacturing and assembly are discussed. The main structure of ship oily water dynamic hydrocyclone separator and its working principle are introduced. The basic parameters of the device working conditions are provided. The new method onboard ship oily water treatment is put forward. It can be used as references for researchers on oily water disposal.
\end{abstract}

Keywords: Dynamic Hydrocyclone, Bilge Water, Structure Design

\section{Introduction}

There are two ways of producing bilge water on board, one is from the maintenance, leakage in engine room; the other is from ballast water in oil tank, cleaning water in ballast tank and washing oil tank. For one 10000GT vessel, the former quantity is small, about every 2-3 tons one day; The latter quantity is big, usually hundreds of tons each time. According to the requirements of the MARPOL 73/78 convention, it must be installed bilge water treatment plant on board, and the oil content in discharged water from ship to the sea through separator must not exceed 15 PPM[1]. "Gravity - coalescence combination" and "filter - coalescence combination" oily-water separator are mostly installed on ship at present. The separation accuracy can meet the requirements of MARPOL73/78 convention, but its processing flow and convenience of operation should in need of improvement. Oily water dynamic hydrocyclone separator was developed by our scientific research team.

Through the prototype experiment shows that the separation accuracy through the oily water dynamic hydrocyclone separator with auxiliary filter adsorption method can meet the requirements of MARPOL73/78 convention and the amount of separation and convenience of operation can be improved well. Oily-water separation effect is satisfied.

\section{The Structure and Working Principle}

\subsection{The Main Structure}

Dynamic hydrocyclone separator is made up of oily water inlet 、 rotary bowl、water outlet and oil outlet and other parts, its structure design as shown in figure 1. It is important that the alignment of the bowl and the two support bracket. So the two support bracket are designed to install on the same high orientation precision of the slide way. It is good for installation and disassembly maintenance, and easy to realize the different size parameters test. The brackets are made of high intensity I-beams in order to enhance its rigidity and reduce the vibration of the equipment. The rotary bowl is the main part of oily water dynamic hydrocyclone separator at the separation zone. Cyclone separation of high-speed rotation of the separated liquid in the bowl is driven by external power (motor), separation effect of gain is accomplished by external force, the pressure loss is very small and easy to realize at low pressure operation; The high-speed rotation of liquid in static 
hydrocyclone is provided by liquid itself, it needs to have enough inlet pressure, so the separation efficiency is obtained through the pressure loss of fluid itself to accomplish, the pressure loss is relatively large [2].

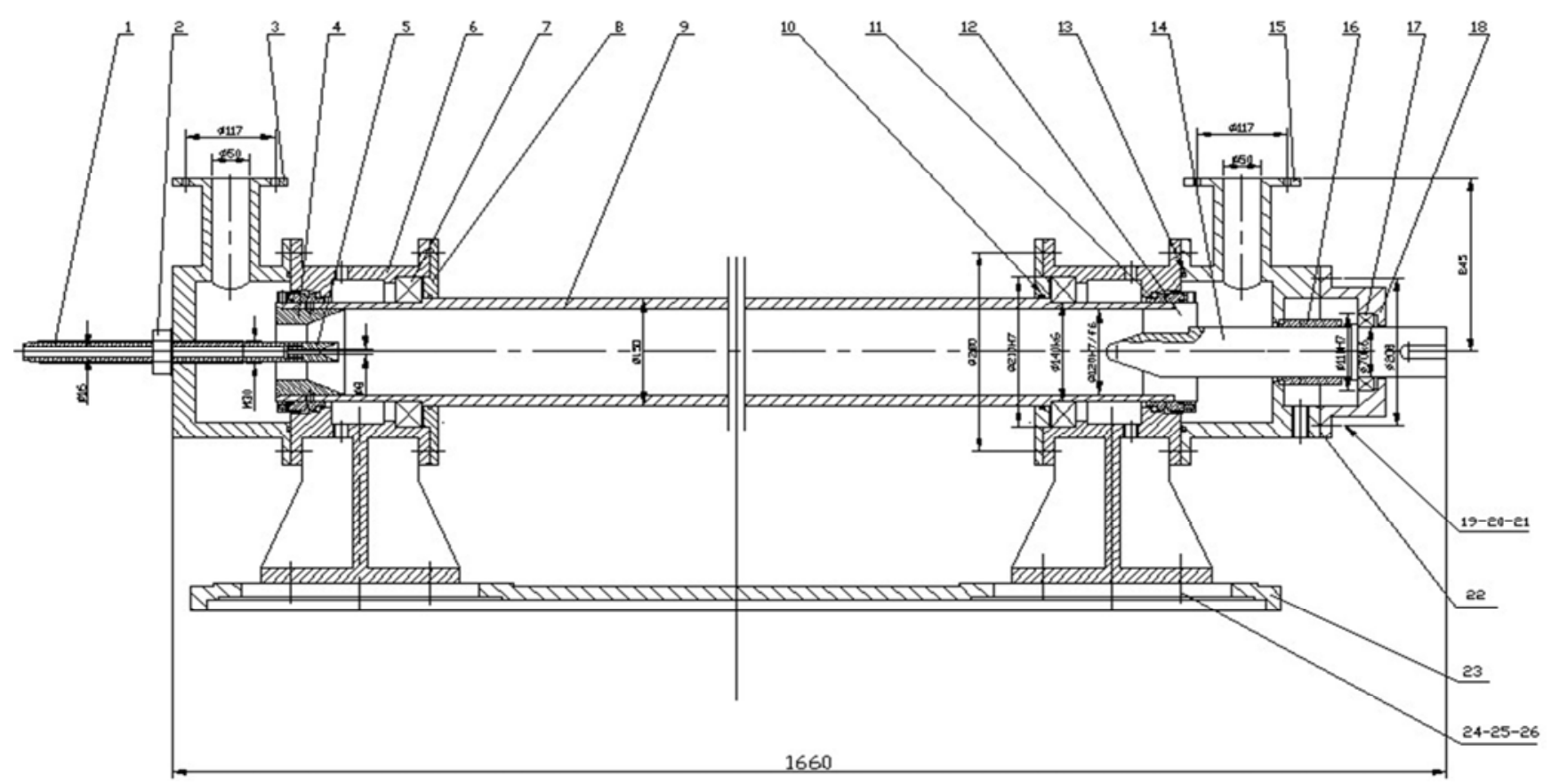

Figure 1. Oily water dynamic hydrocyclone separator structure.

1-overflow tube2-lock nut 3-shell cover 4-bottom outlet flow cone 5-overflow nozzle 6-support 7-bearing 8-bearing cap 9-rotary bowl 10- O sealing 11-mechanical seal 12-grid sheet 13-O sealing 14-shaft 15-inlet cover 16-mechanical seal 17-bearing 18-inlet auxiliary cover 19-stud 20-nut 21-washer 22-gasket 23-foundation base 24-stud 25-nut 26-washer

\subsection{The Basic Principle of Dynamic Hydrocyclone}

The basic principle of dynamic hydrocyclone is the use of density difference between oil and water in oily-water mixture liquid to obtain the centrifugal separation. The centrifugal force of the oil and water are different due to the density difference between oil and water when oily water mixture in the high-speed rotary, The obtained centrifugal force of the water is bigger than the oil, so the water is gathered at the periphery of the rotary bowl; the oil is gathered at the center of the rotary bowl, then the water and oil are separated from the oily water mixture [3]. It is much more difficult in the oil/water separation than solid-liquid separation because the density difference between the oil-water mixture liquid is often smaller, generally only $0.1 \sim 0.2 \mathrm{~g} / \mathrm{cm}^{3}$. Secondly the oil exists in the form of droplets in oily-water mixture; it is easy to deformation in stress and even rupture, it is very important for Oil-water separation to form a strong vortex and to prevent the crushing of the oil droplets.

As shown in figure 1. Rotary bowl is driven through belts by motor to obtain high speed rotation, oily-water mixture is transferred at the entrance by the centrifugal pump, oily-water mixture is pressed into the way to the rotating grid sheets, and through the rotating shaft guide cone into the vortex chamber. Oily-water mixture through the rotating grid sheets mainly enhances rotation acceleration, followed by axial guiding. Oily-water mixture with high-speed rotation depends on the friction of the rotary bowl wall formed the larger, more strong eddy velocity field, the motion curve of the particle in mixture is screw type, known as the "rotational flow". High-speed rotation of the oil-water mixture depends on the density difference, low density oil is pressed to drum center and formed oil core; Heavy density water towards to the rotary cylinder wall; At the same time, liquid flow moves to the other end of the rotating drum by axial force (pressure). Oil in the center is taken in the overflow mouth and the oil outlet, and water at rotating cylinder wall s discharged through outlet of water. Last the oil and water are separated.

\section{The Structure Design}

Due to the fine filter adsorption is already relatively mature; our research team put the key research content to the rotating barrel dynamic hydrocyclone structure and the relationship between the parameters and the separation effect.

\subsection{Rotating Cylinder Diameter D and Length L}

The cyclone separation efficiency depends on the strength of the rotating cylinder internal flow field, and the strength of the vortex flow field depends on the inner diameter of the drum size; At the same time, its effect also depends on the liquid residence time within the rotating drum, and residence time depends on the length of the rotary bowl. The larger rotating bowl diameter $D$ is, the greater tangential velocity is, the bigger centrifugal force of separation is, and will be beneficial to oil-water separation; The longer bowl length $L$ is, 
the greater the oil-water resides in the bowl is, the separation effect will be better. So, the length of the rotating bowl should be long enough in order to ensure the liquid resides in the bowl[4],[5],[6]. Normally reside time should be in $3 \sim 10$ s. $D$ and $L$ of the rotary bowl affect the capacity of dynamic hydrocyclone, the greater the capacity is, the longer of the separation time should be. Assume reside time is $t$, then the quantity $Q$ is:

$$
\mathrm{Q}=\left(9 \times 10^{-7} \pi \mathrm{D}^{2} \mathrm{~L}\right) / \mathrm{t}
$$

Rotary bowl which rotating at high speed is an important part of a dynamic hydrocyclone separator, it is important to its strength and dynamic balance. In order to avoid intensity drops and reducing the dynamic balance destruction phenomenon by welding, the standard circular steel pipe is used to make rotary bowl. After processing, static and dynamic balance should be tested in order to ensure the rotation accuracy required by the rotating bowl.

\subsection{Rotary Grid Structure Design}

At the entrance of the mixed liquid into the rotary bowl, rotary grids which make the mixture form stable fluid vortex in the rotary bowl are installed. The rotary grids will cause resistance and pressure loss by the flow of the mixture, the higher the rotational speed is, the bigger pressure loss is; the quantity of rotary grid e will directly proportional to vortex strength of the fluid, also increase the pressure loss [7]. According to The dynamic hydrocyclone structure parameters optimization design [8], three pieces rotary grids are installed in rotary bowl in order to improve the separation efficiency, easy processing, convenient installation and other elements to the common principles. After calculated, the rotary grids length about70 $\mathrm{mm}$ is considered the most appropriately. At the same time, an optimization design of the rotary grids which have the effect of rotary bowl transmission torque is used to select the grid material, leaf thickness and depth of the leaf inlay groove.

\subsection{The Overflow Mouth Structure and Parameters}

One function of the overflow mouth is to collect and discharge the separated oil at the center of the rotary bowl by the rotation of the oily water mixture; the other is to separate water and oil from oil water interface effectively near the overflow mouth [9]. If the inner diameter of the overflow mouth is too big, water will be in the separated oil; otherwise, oil will be in the separated water with oil. The overflow mouth is stuck into rotary bowl too deep or too shallow, water will be in the separated oil too [10]. So the axial location and diameter of the overflow mouth should be matched to the terminal position and the diameter of the separated in the bowl. Therefore, there are three different diameters $(8 \mathrm{~mm}, 10 \mathrm{~mm}$, $12 \mathrm{~mm}$ ) of the overflow mouth which axial position can be adjusted by rotating the overflow tube with a fine thread.

\subsection{Bottom Outlet Flow Cone}

Bottom outlet flow cone is the convergent type which is good for the flow field of discharge oil at the outlet end and making the axial position of the overflow mouth convenient adjustment. The stable vortex flow field will not to be destroyed easily in the rotary bowl if $t$ as small as possible at bottom outlet flow cone, the transition taper angle is selected 20 degrees.

\subsection{Alignment of Rotary and Pedestal}

Alignment of rotary bowl and pedestal is one of the important factors to affect the rotational stability which is conducive to form stable oil core and to discharge oil through the discharge port, so, rotate smoothly or not directly affect the cyclone separation efficiency. If the alignment accuracy is not high, the intense vibration of the equipment can cause oil core diameter become coarse and can't even produce oil core. In addition, the alignment not only affects the stability of rotary bowl rotation, but also affects the sealing performance of the equipment. Therefore, the basic size and tolerance of two pedestals are the same data, and mechanical shaft seals with good performance and not high dimensional accuracy requirements are selected at the ends of the rotary bowl is. It can ensure the sealing performance, and can reduce power consumption and improve the service life.

\subsection{Power Transmission System}

The function of power transmission system is to transfer the motor torque to the rotary bowl and drive the rotary bowl rotation at a certain speed. Transmission mode can be bear or $\mathrm{V}$ belt drive, $\mathrm{V}$ belt transmission have end $\mathrm{V}$ belt wheel transmission and rotary bowl with v-belt itself. The rotary bowl end $\mathrm{V}$ belt wheel transmission is used in our design due to considering the factors such as manufacturing cost, convenient maintenance process.

\section{Parts Manufacture and Matching}

\subsection{Foundation Base}

Foundation base is the basis of supporting dynamic hydrocyclone separator, it is smooth or not that directly affect the alignment precision of equipment, and the separation efficiency. To meet the above requirements, the foundation base is processed by the planer in the whole piece of channel steel in order to ensure the foundation base size precision. The foundation base and two support base pedestals are clamped and processed at the same time on boring machine to ensure alignment.

\subsection{Assembly}

The dynamic hydrocyclone separator performance depends not only on the manufacturing precision of components, but also depends on the assembly precision. The radial run out of rotary bowl shall not be greater than $0.1 \mathrm{~mm}$, it is very difficult For this reason, the measurement and installation are carried on the unification of datum to the base plate on the foundation base both to ensure accuracy and convenient installation. 


\subsection{Motor}

The quantity of bilge water is changing every day by dynamic hydrocyclone separator on board, it can be solved by adjusting the separator working hours if the variation is small, but if the variation is larger, only by adjust the working hours is hard to work, it can be solved by increasing the speed of the rotary bowl. The motor should be selected step speed multi-polar logarithmic ac asynchronous motor or step less speed frequency conversion motor.

\section{Field Experiment}

The oil content of bilge water from engine room mainly consists of fuel oil and lubrication oil. The oil concentration can reach $1000 \mathrm{mg} / \mathrm{L}$. The total amount is about $3-5 \mathrm{t} / \mathrm{d}$. The oily water from cargo oil tank contains ballast water and washing water. Its main constituents are crude oil, fuel oil and lubrication oil. The oil concentration is $2000 \mathrm{mg} / \mathrm{L}-7000 \mathrm{mg} / \mathrm{L}$ and the total amount is hundreds to thousand tons per time.

In view of the practical situation, the experiment takes oily water with concentration of $2000 \mathrm{mg} / \mathrm{L}$ as the example. The ambient temperature is $2^{\circ} \mathrm{C}-30^{\circ} \mathrm{C}$. The speed, the output speed of common three-phase asynchronous motor, is $1300 \mathrm{r} / \mathrm{min}-1450 \mathrm{r} / \mathrm{min}$. Set the input pressure at $0.25 \mathrm{Mpa}$. Select $10 \mathrm{~mm}$ overflow vent. Adjust the back pressure P of outlet and perform the experiment on the variation trend of back pressure $\mathrm{P}$ of outlet, oil concentration $\mathrm{C}$ of discharge and capacity Q. The corresponding relationship is shown in Table 1. The corresponding relationship curve of back pressure $P$ of outlet and oil concentration $\mathrm{C}$ of discharge is shown in Figure 2. The corresponding relationship curve of back pressure $P$ of outlet and capacity $\mathrm{Q}$ is shown in Figure 3. In view of the required disposal capacity, $2.0 \mathrm{M}^{3} / \mathrm{h} \sim 3.0 \mathrm{M}^{3} / \mathrm{h}$, onboard ship, the back pressure should varied from $0.04 \mathrm{Mpa}$ to $0.06 \mathrm{Mpa}$. In this way, it can meet the requirements of disposal capacity and decrease the oil concentration below $20 \mathrm{mg} / \mathrm{L} \sim 40 \mathrm{mg} / \mathrm{L}$. Under the help of following filtering and adsorbing elements, it can meet the requirements of MARPOL73/78 Convention, 15PPM. When the back pressure is lower than $0.08 \mathrm{Mpa}$, the oil concentration also can be less than 15PPM, but the capacity is not satisfying in practical operation. Therefore, it is inadvisable.

Table 1. The corresponding relationship of back pressure $P$, oil concentration $C$ and capacity $Q$.

\begin{tabular}{|c|c|c|c|c|c|c|c|c|c|}
\hline \multirow[b]{2}{*}{ Back Pressure $\boldsymbol{P}$ (MPa ) } & \multicolumn{9}{|c|}{ Experiment Results } \\
\hline & 0.00 & 0.02 & 0.04 & 0.06 & 0.08 & 0.10 & 0.12 & 0.14 & 0.16 \\
\hline Oil Concentration $C(\mathrm{mg} / \mathrm{L})$ & 120 & 80 & 40 & 20 & 15 & 12 & 10 & 9 & 8 \\
\hline Capacity $Q\left(\mathrm{M}^{3} / \mathrm{h}\right)$ & 4.0 & 3.8 & 2.8 & 2.0 & 1.0 & 0.7 & 0.5 & 0.4 & 0.3 \\
\hline
\end{tabular}

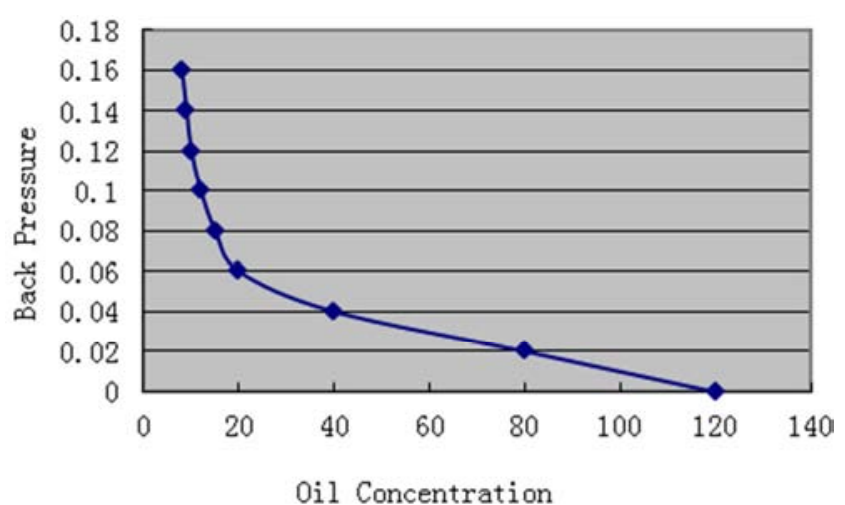

Figure 2. The corresponding relationship curve of back pressure $P$ and oil concentration $C$.

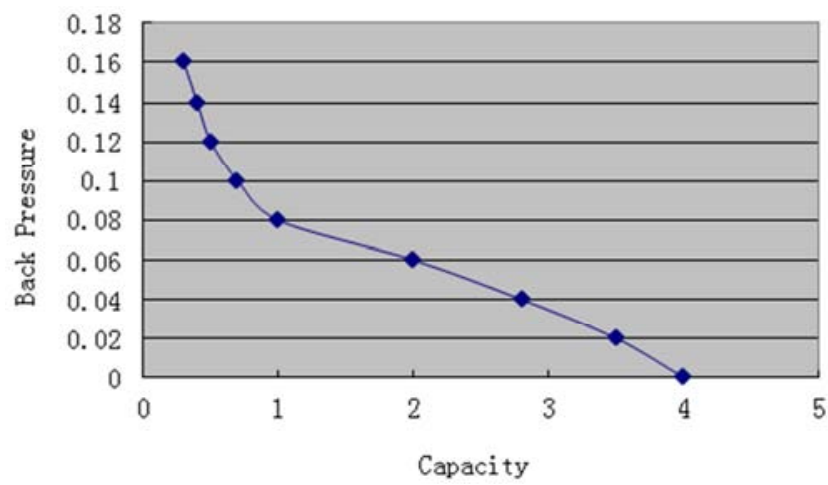

Figure 3. The corresponding relationship curve of back pressure $P$ and capacity $Q$.

\section{Conclusions}

Fuel oil and lubricating oil are main components in the bilge oily water on ship engine room, the oil concentration is over $1000 \mathrm{mg} / \mathrm{L}$. Integrated actual situation, field test was carried out at 20 to $26^{\circ} \mathrm{C}$ environment temperature used the oil (fuel oil and lubricating oil) concentration of $2000 \mathrm{mg} / \mathrm{L}$ of the oil-water mixture separation, outlet oil content in water can be dropped to $20 \mathrm{mg} / \mathrm{L}$ at water outlet, and the subsequent secondary filtration adsorption treatment method can meet the requirements of 15 PPM MARPOL73/78 convention.

Separation effect of oily water by dynamic hydrocyclone separator depends on multiple correlation factors, such as separator geometry size, working temperature, concentration of oil, oil varieties, inlet pressure, back pressure, rotation speed, quantity of the bilge water, and so on[11]. According to existing geometry size (as shown in figure 1), the optimal rotate speed is $1300 \mathrm{RPM}$, the inlet pressure is $0.45 \mathrm{MPa}$, the outlet pressure is atmospheric pressure and flow is $3 \mathrm{~m}^{3} / \mathrm{h}$, tested with concentration of $2000 \mathrm{mg} / \mathrm{L}$ oil (fuel oil and lubricating oil) and water mixture in the dynamic hydrocyclone separator, test results is satisfactory. But emulsion, laminar and turbulent flow, viscous, resonance, the inner surface roughness and other deep-seated problems have not involved, remains to be further discussed in the future.

Dynamic hydrocyclone separation, in short, is an emerging technology, both in the equipment structure parameters optimization, the separation mechanism of research, or 
operational parameters and structure parameters of the studies are subject to a common relationship

\section{References}

[1] Li Pinfang. Ship management [M]. Dalian: dalian maritime university press, 2008.6.

[2] M. Piller, G. Schena, G. Belardi. Sensitivity of DynaWhirlpool hydrocyclone operation toapplied back-pressure [J]. International Journal of Mineral Processing, v 154, p 81-93, September 10, 2016.

[3] Lv Fengxia, Wang Zunce, etc. Experimental study on dynamic hydrocyclone for thin oil dewatering $[\mathrm{J}]$. Proceedings of the International Conference on Offshore Mechanics and Arctic Engineering - OMAE, v 4, n PART A, p 35-41, 2009.

[4] Caie Zhang, Dezhou Wei, etc. Effects of curvature radius on separation behaviors of the hydrocyclone with a tangent-circle inlet [J]. Powder Technology, v 305, p 156-165, January 1, 2017.

[5] Reza Sabbagh, Michael G. Lipsett, etc. Predicting equivalent settling area factor in hydrocyclones; a method for determining tangential velocity profile $[\mathrm{J}]$. Separation and Purification Technology, v 163, p 341-351, May 11, 2016.
[6] Vieira, Luiz G,M. Silva, Danylo O, etc. Effect of Inlet Diameter on the Performance of a Filtering Hydrocyclone Separator. Chemical Engineering and Technology, v 39, n 8, p 1406-1412, August 1, 2016.

[7] Li, Gao-Sen, Chen,Jia-Wang, etc. The effect of the pressure drop ratio on the performance of a novel dynamic hydrocyclone [J]. Applied Mechanics and Materials, v 496-500, p 827-830, 2014.

[8] Wang Zunce, Chen Weiqin,Jiang Minghu, etc. The optimization design of dynamic hydrocyclone structure parameters [J]. Acta Petrolei Sinica, 2001, 22 (4): 104-107.

[9] Ma, Qiang, Miao, Yuna, etc. Design of ship dynamic hydrocyclone slop treatment system [J]. Proceedings 2010 IEEE International Conference on Information Theory and Information Security, ICITIS 2010, p 697-700, 2010.

[10] Chandranath Banerjee, Eric Climent, Arun Kumar Majumder. Mechanistic modelling of water partitioning behaviour in hydrocyclone $[\mathrm{J}]$. Chemical Engineering Science, v 152,p 724-735, October 2, 2016.

[11] Petar Durdevic, Simon Pedersen, Zhenyu Yang. Evaluation of OiW Measurement Technologies for Deoiling Hydrocyclone Efficiency Estimation and Control [J]. OCEANS 2016 Shanghai, June 3, 2016, OCEANS 2016 - Shanghai. 